\title{
Towards green frameworks for digital forensics investigation
}

\begin{abstract}
Despite the fact that digital forensics involves strict procedures and complies with fixed regulations and principles, but as this paper presents, there are plenty of opportunities that can be practically employed in digital forensics to make this science greener. Virtualization can cost effectively reduce the number of workstations running forensic tools in the lab. Cloud computing and consolidating servers and storage devices in green data centers not only facilitate managing and securing services but also decline the number of required network and cooling facilities. Forensic labs can also be optimized with regard to environmental preservation. Using remote protocols and digitalizing paperwork procedures are environmentally helpful practices to accelerate investigation progress as well. Improving electrical power needs of labs and forensic devices is another issue that should be taken into consideration. Employing storage devices with optimal energy usage in digital forensics may highly reduce energy consumption. This paper study established green technologies particularly in information technology field and suggests a framework for implementing compatible techniques in digital forensics in order to reduce greenhouse gas pollutants, limit carbon emissions, and preserve the environment.
\end{abstract}

Keyword: Green forensics; Digital forensics; Environmental preservation; Forensic lab 